\title{
Das LEaD-Kompetenzmodell - wirksam Führen im Kontext der digitalen Transformation
}

\author{
Stefan L. Dörr' • Marion Schmidt-Huber' · Günter W. Maier²
}

Angenommen: 29. April 2021 / Online publiziert: 10. Juni 2021

(c) Der/die Autor(en) 2021

\section{Zusammenfassung}

Dieser Beitrag der Zeitschrift Gruppe. Interaktion. Organisation. (GIO) stellt die Entwicklung, empirische Validierung und Anwendung des LEaD-Modells ${ }^{\circledR 1}$ (Leadership Effectiveness and Development) der Führung im Rahmen der digitalen Transformation vor. Die Auswahl und Entwicklung von Führungskräften und die dazu erforderliche evidenzbasierte Diagnostik von Führungskompetenzen stellen zentrale Herausforderungen einer modernen HR-Strategie dar, wie z.B. beidhändige Führung im Kontext der digitalen Transformation und entwicklungsorientierte Diagnostik. Im Kontext des digitalen Wandels ist „LEaD“ ein Instrument für Führungskräfte und HR-Spezialist*innen, um Transformationsprozesse durch gezieltes Kompetenzmanagement erfolgreich zu bewältigen und eine neue Führungskultur zu etablieren. Praktische Anwendungsbereiche des LEaD-Modells in Organisationen sind sowohl auf individueller (z. B. als Führungsfeedback mit Selbst- und Fremdeinschätzung) als auch organisationaler Ebene (z. B. Messung der Führungseffektivität) möglich.

Dieser Beitrag beschreibt das LEaD-Modell der Führung als einen evidenzbasierten Handlungsrahmen für Führungskräfte, der Diagnose- und Interventionsmaßnahmen bietet und damit einen wichtigen Beitrag zu einer nachhaltigen und lohnenswerten Investition in Führung leistet.

Schlüsselwörter Führung · Digital Leadership · Digitale Transformation · Veränderungen in der Arbeitswelt · Change Management $\cdot$ Kompetenzmanagement $\cdot$ Kompetenzen $\cdot$ Kompetenzmodelle $\cdot$ Ambidextrie $\cdot$ Beidhändige Führung LEaD-Modell $\cdot$ Managementdiagnostik $\cdot$ Führungsentwicklung und -training $\cdot$ Führungsforschung $\cdot$ Evidenzbasiertes Management

Beim LEaD-Modell@ handelt es sich um ein geschütztes Kompetenzmodell von A47-Consulting, das zur einfacheren Lesbarkeit im Folgenden ohne Copyright-Kennzeichnung und als LEaD-Modell bzw. als LEaD bezeichnet wird.

Dr. Stefan L. Dörr

stefan.doerr@a47-consulting.de

Dr. Marion Schmidt-Huber

marion.schmidt@a47-consulting.de

$\triangle$ Prof. Dr. Günter W. Maier

ao-psychologie@uni-bielefeld.de

1 A47-Consulting Unternehmensentwicklung \& Managementdiagnostik, Agnesstraße 47, 80798 München, Deutschland

2 Fakultät für Psychologie und Sportwissenschaft, Arbeitsund Organisationspsychologie, Universität Bielefeld, Universitätsstraße 25, 33615 Bielefeld, Deutschland 


\title{
The LEaD competence model: Leading effectively in the context of digital transformation
}

\begin{abstract}
This contribution of the magazine Group. Interaction. Organization. Journal of Applied Organizational Psychology (GIO) presents the development, empirical validation, and application of the LEaD-Model ${ }^{\text {I1 }}$ (Leadership Effectiveness and Development) of leadership in the context of digital transformation. The selection and development of leaders and the evidence-based diagnostics of leadership competencies required for this represent central challenges of a modern HR strategy, such as ambidextrous leadership in the context of digital transformation and development-oriented diagnostics. In the context of digital transformation, LEaD is a tool for managers and HR specialists to successfully manage transformation processes through targeted competence management and to establish a new leadership culture. Practical applications of the LEaD model in organizations are possible at both the individual (e.g., as leadership feedback with self-assessment and peer assessment) and organizational levels (e.g., measuring leadership effectiveness).

This paper describes the LEaD model of leadership as an evidence-based framework for action by leaders that provides diagnostic and intervention measures and thus makes an important contribution to a sustainable and worthwhile investment in leadership.
\end{abstract}

Keywords Digital leadership - Leadership culture - Digital transformation - New work design $\cdot$ Change management . Competencies · Competence management - Competency models · Management diagnostics · Leadership development . Leadership training $\cdot$ Leadership research $\cdot$ Ambidexterity $\cdot$ Ambidextrous management $\cdot$ Evidence-based management

\section{Führung als wichtiger Erfolgsfaktor in der digitalen Transformation}

Welche Rolle spielt Führung für den Unternehmenserfolg in Zeiten der digitalen Transformation? Digital Leadership (Dörr et al. 2018) umfasst moderne Konzepte der Führung, die neue Anforderungen an Führung im digitalen Wandel beschreiben. Welpe et al. (2018) formulieren mit den „Big 5 des Digital Work Designs" Kompetenzen, die mit einer neuen Führungskultur einhergehen, welche sich unter anderem durch mehr Offenheit, Vertrauen, Kollaboration und Gesundheitsorientierung auszeichnet und zentrale Erfolgsfaktoren darstellen, dass Unternehmen in einer globalen, dynamischen und zunehmend vernetzten Welt die notwendige Agilität und Adaptionsfähigkeit entwickeln (vgl. Kauffeld und Maier 2020). Die Anforderungen an Führungskräfte, die sich durch die digitale Transformation ergeben, wie z.B. Umgang mit neuen Technologien, mehr Team- und Vertrauenskultur, geteilte Führung und stärkere vernetzte Zusammenarbeit, sind aber nicht völlig neu. Führungsansätze wie authentische, transformationale oder charismatische Führung haben längst gezeigt, dass Führungsverhalten, das Mitarbeitende inspiriert, empowert und Vernetzung fördert, zu mehr Vertrauen und Identifikation mit der Führungskraft (Nübold et al. 2015) und letztlich zu höherer Leistung im Team führt (vgl. Lord et al. 2017). Für Führungskräfte kommt es darauf an, in der digitalen Transformation die richtige Balance zwischen Kerngeschäft und Innovation zu finden. Dabei spricht man von „,beidhändiger Führung“ (Petry 2016) oder Ambidextrie (vgl. O'Reilly und Tushman 2013). Wie gelingt es Unternehmen, ihre Führungskräfte für den anstehenden Transformationsprozess der Digitali- sierung fit zu machen? Das in den nachfolgenden Kapiteln näher beschriebene LEaD-Modell der Führung ist ein Rahmenmodell und unterstützendes Werkzeug bei der Auswahl und Entwicklung von Führungskräften und gibt mit seinen 18 Dimensionen wertvolle Hinweise für ein situationsangemessenes Agieren der Führungskräfte in verschiedenen Kontexten, wie auch insbesondere dem der digitalen Transformation.

\section{Konzeption, Entwicklung und Validierung des LEaD-Kompetenzmodells der Führung}

Mit der Entwicklung des LEaD-Modells wurde das Ziel verfolgt, ein universelles, evidenzbasiertes und zugleich praxisorientiertes, flexibel anwendbares Kompetenzmodell für die Führungskräfteauswahl und -entwicklung zu konzipieren. Universell bedeutet, dass es für Führungspositionen auf unterschiedlichen Hierarchiestufen und in unterschiedlichen Branchen eingesetzt werden kann. Flexibel bedeutet in diesem Zusammenhang, dass es als grundlegendes Modell konzipiert ist, das an die spezifischen Anforderungen in Unternehmen angepasst werden kann. Evidenzbasiert und praxisorientiert bedeutet für den Prozess der Kompetenzmodellierung und die Instrumentenentwicklung, ein wechselseitiges praxis- und wissenschaftsorientiertes Vorgehen zu wählen, orientiert an der rationalen Konstruktionsstrategie (Fisseni 2004) und den Anforderungen der DIN 33430 an berufsbezogene Eignungsdiagnostik (Westhoff et al. 2005).

Der zugrundeliegende Kompetenzbegriff im LEaD-Modell bezieht sich auf ein verhaltensnahes und performanceorientiertes Verständnis von Kompetenz, definiert als Fä- 
higkeiten und Verhaltensweisen einer Führungskraft, die zur erfolgreichen Bewältigung von Führungsaufgaben erforderlich sind (Campion et al. 2011; Schippmann et al. 2000). Dem LEaD-Modell liegt die Annahme zugrunde, dass über verschiedene Branchen, Hierarchieebenen und Tätigkeiten hinweg grundlegende Gemeinsamkeiten in den Anforderungen der Führungspositionen bestehen, die im Rahmen von Kernkompetenzen abgebildet werden können (vgl. Schippmann et al. 2000) Im Folgenden wird zunächst das LEaD-Modell im Überblick beschrieben. In den weiteren Abschnitten werden die Entwicklung und theoretische Ableitung sowie die empirische Überprüfung und Validierung von „LEaD“ vorgestellt.

\subsection{Beschreibung des LEaD-Modells}

„LEaD“ bietet einen evidenzbasierten Handlungsrahmen für effektives Führungshandeln (vgl. Dörr et al. 2012, 2016, 2017b; Schmidt-Huber et al. 2014). Im Modell werden fünf übergeordnete Kompetenzfelder (mit jeweils einem Beispielitem) als zentrale Anforderungen an Führungskräfte beschrieben:

1. Strategieorientierung (Chancen erkennen, Zukunftsperspektive formulieren, Innovationen fördern): Die Führungskraft richtet ihr Denken und Handeln klar an den Anforderungen des Markts und der Kundenbedürfnisse aus und stellt übergreifende Sinnzusammenhänge her. Eine zukunftsorientierte strategische Perspektive vermittelt Mitarbeitenden Orientierung, indem das „Big Picture“ aufgezeigt und der Fokus auf die Marktanforderungen und Kundenbedürfnisse gerichtet wird. Es entsteht Motivation durch Sinnstiftung.

Beispielitem: Die Führungskraft berücksichtigt Kundenbedürfnisse und Trends bei der Entwicklung ihrer/seiner Strategie.

2. Ergebnissicherung (Ziele vereinbaren, Probleme analysieren, Ergebnisse bewerten): Die Führungskraft leitet in Kooperation mit den Mitarbeitenden herausfordernde Ziele ab, analysiert Problemstellungen, definiert Verantwortlichkeiten, initiiert Umsetzungsmaßnahmen und überprüft regelmäßig die Ergebnisse. Motivation entsteht durch Handlungsorientierung, Selbstwirksamkeit, Ergebnisorientierung und konkrete Erfolgserlebnisse.

Beispielitem: Die Führungskraft vereinbart beidseitig getragene Ziele.

3. Mitarbeiter-/Teamentwicklung (Verantwortung delegieren, Feedback geben, Mitarbeitende coachen, Perspektiven übernehmen): Die Führungskraft entwickelt und fördert einzelne Mitarbeitende und ganze Teams in ihren Kompetenzen und unterstützt sie dabei, konstruktiv zusammenzuarbeiten, ihre Ziele zu erfüllen und sich persönlich bzw. als Team weiterzuentwickeln. Sie motiviert durch persönliche Wertschätzung, Coaching und individuelle Förderung der Kompetenzen, Feedback und dem Aufzeigen individueller Entwicklungsperspektiven.

Beispielitem: Die Führungskraft hilft ihren/seinen Mitarbeitern Kompetenzdefizite zu erkennen und sich weiterzuentwickeln.

4. Umfeldgestaltung (Effektiv kommunizieren, Ressourcen bereitstellen, Konflikte managen, Veränderungen umsetzen, Beziehungen managen): Die Führungskraft ist sich der Veränderungen in ihrem Umfeld bewusst und gestaltet den Kontext aktiv durch die Etablierung von Kommunikationsprozessen, durch effektives Ressourcenmanagement, die Initiierung von Konfliktlösungen und durch gezieltes Networking. Dadurch kreiert sie die Rahmenbedingungen, die für ein effektives und motiviertes Arbeiten notwendig sind.

Beispielitem: Die Führungskraft sorgt bei einem Veränderungsprozess für das Sichtbarwerden erster Erfolge.

5. Persönliche Einflussnahme (Selbstvertrauen vermitteln, Authentizität ausstrahlen, Ambiguitäten managen): Die Führungskraft überzeugt und motiviert durch persönliche Integrität, sie setzt vielseitige soziale Einflussstrategien situationsangemessen ein und vermittelt ihren Mitarbeitenden Selbstvertrauen. Bei ihrer sozialen Einflussnahme legt sie Wert auf ein ,,authentisches Vorbildverhalten“. Sie ist sich der verschiedenen Spannungsfelder und Ambivalenzen des Führungsalltags bewusst, geht mit diesen lösungsorientiert um und handelt reflektiert und gestaltend.

Beispielitem: Die Führungskraft macht klar wie wichtig es ist, sich hundertprozentig für eine Sache einzusetzen.

\subsection{Theoretische Ableitung erfolgskritischer Kompetenzen}

Die Vielzahl empirischer Erkenntnisse und daraus resultierenden Handlungsempfehlungen im Feld der Führungsforschung in den letzten 25 Jahren - Hiller et al. (2011) berichten z.B. von 1161 veröffentlichten wissenschaftlichen Arbeiten - erweisen sich für die Führungspraxis als zu unübersichtlich und mit häufig zu hoher Spezifität. In der Führungsforschung werden beispielsweise die vier Stränge der eigenschaftsorientierten, verhaltensorientierten, situativen sowie charismatischen/transformationalen Ansätze voneinander unterschieden (vgl. im Überblick: Braun et al. 2017). Darüber hinaus finden in den vergangenen Jahren weitere Ansätze, wie z.B. zur strategischen und authentischen Führung, eine verstärkte Aufmerksamkeit: Aufgrund der markt- und wettbewerbsbedingten Veränderungen in Organisationen rücken die strategischen Kompetenzen von Führungskräften (z. B. Boal und Hooijberg 2001) stärker in den Fokus. Im Rahmen neuerer Ansätze, dem sogenann- 
ten „New Leadership Approach“ (vgl. Felfe 2015), wird darüber hinaus stärker das integrative Zusammenspiel personaler, situativer, verhaltensbezogener und transformationaler Kompetenzelemente hervorgehoben (vgl. Antonakis und House 2004; Dörr 2008).

Diese Dichte an Ansätzen und Theorien erschwert die Ableitung von anwendungsnahen Empfehlungen zu evidenzbasierter Führungsdiagnostik und -entwicklung. Aus diesem Grund wurden für die Entwicklung der LEaD-Modellinhalte wissenschaftliche Führungstheorien gesichtet, die inhaltlich relevantesten sowie hinsichtlich verschiedener leistungs- und einstellungsbezogener Kriterien erfolgswirksamsten Führungskonstrukte ausgewählt und in inhaltsnahe Kategorien überführt. Diese Inhaltscluster bilden die 5 übergeordneten Kompetenzbereiche des LEaD-Modells ab: 1) Strategieorientierung, 2) Ergebniserreichung, 3) Mitarbeiter-/Teamförderung, 4) Umfeldgestaltung und 5) Persönliche Einflussnahme. Zur theoriebasierten Operationalisierung des Modells wurden daraufhin die Inhalte der inkludierten Führungstheorien den übergeordneten Kompetenzbereichen zugeordnet (vgl. z. B. Campion et al. 2011). So wurde beispielsweise Strategieorientierung mit den Dimensionen „Chancen erkennen“, „Zukunftsperspektiven formulieren und Innovationen treiben" operationalisiert (Überblick zur Theorieverortung siehe Schmidt-Huber et al. 2014).

\subsection{Praxisbezogene Erweiterung des LEaD-Modells:}

Die Praxisrelevanz der fünf theoriebasierten LEaD-Kompetenzbereiche wurde durch eine qualitative Anforderungsanalyse mit Führungskräften verschiedener Hierarchiestufen überprüft (Schmidt-Huber et al. 2014). Mit BehavioralEvent-Interviews (McClelland 1998) wurde der Anforderungsbezug der fünf Kompetenzbereiche validiert: Welchen erfolgskritischen Führungssituationen begegnen Führungskräften aus unterschiedlichen Funktionen, Hierarchieebenen und Verantwortungsbereichen? Welche Kompetenzen erweisen sich - aktuell und in Zukunft - als erfolgskritisch und wirksam zur Bewältigung der Herausforderungen?

Die Auswertung der Interviews erfolgte durch eine strukturierte Inhaltsanalyse. Von Fachexpert*innen (Arbeits- und Organisationspsycholog*innen, langjährige Managementberater*innen) konnten $83 \%$ der gesammelten erfolgskritischen Situationen dem LEaD-Modell zugeordnet werden. Aus den verbliebenen $17 \%$ Führungssituationen wurde auf praxisrelevante Lücken im LEaD-Modell geschlossen (z. B. die Identifikation von Problemen). Hierfür wurden weitere Modelldimensionen, orientiert an empirisch relevanten Taxonomien von Führungskompetenzen (Borman und Brush 1993; Fleishman et al. 1991; Tett et al. 2000), gebildet. Das nunmehr theoriebasierte und praxisorientierte und im ersten Schritt erprobte Kompetenzmodell wurde in drei weiteren Studien empirisch überprüft, um ein valides und reliables Messinstrument zu entwickeln und gleichzeitig die Konstruktvalidität und prädiktive Validität des Modells zu ermitteln (vgl. Schmidt-Huber et al. 2014).

\subsection{Empirische Überprüfung des LEaD-Modells: Validierung und Gütekriterien}

Zunächst wurde ein umfangreicher Fragenpool mit 315 Items entwickelt, der sich aus den Inhalten der theoretischen Führungsmodelle, den Befunden aus den Behavioral Event-Interviews und aus den Erkenntnissen aus langjähriger Berater- und Diagnosetätigkeit der Autor*innen zusammensetzte.

Anschließend wurden im Rahmen einer Prototypizitätsstudie mit Fachexpert*innen (Arbeits- und Organisationspsycholog*innen) die inhaltlich relevantesten und verständlichsten Items jeder LEaD-Kompetenz selektiert. Hierbei spielte in erster Linie die Passung der Itemformulierung zu den Kompetenzbereichen eine Rolle (hohe Prototypizität). Der Itempool wurde durch dieses Vorgehen auf 208 Items reduziert.

Die darauffolgende psychometrische Überprüfung der Items diente der weiteren Verdichtung des Fragenpools. Hierfür wurde das LEaD-Instrument erstmals im Rahmen einer Online-Studie angewandt. Alle berufstätigen Teilnehmenden beurteilten das Verhalten ihrer Führungskraft auf einer fünfstufigen Skala ( $1=$ Verhalten nie erkennbar bis $5=$ Verhalten regelmäßig, fast immer erkennbar). Die beurteilten Führungskräfte waren in unterschiedlichen Hierarchieebenen tätig (16,5\% im unteren Management, 43,7\% im mittleren Management, 38,8\% im oberen Management). Aus den Ergebnissen der Itemanalyse wurden die 115 Items mit den besten psychometrischen Eigenschaften selektiert (z.B. Strategieorientierung: Chancen erkennen „denkt vorausschauend und informiert sich über zu erwartende Entwicklungen im eigenen Fachgebiet"), bei inhaltskonvergenten Itempaaren wurde das Item mit den günstigeren Kennwerten ausgewählt.

Abschließend wurde eine umfangreiche Validierungsstudie durchgeführt, um die psychometrische Güte des Instruments sowie die Erfolgsrelevanz der Dimensionen (Kriteriumsvalidität) und den Mehrwert des Instruments bei der Aufklärung von Führungserfolg gegenüber bestehenden Führungsinstrumenten (inkrementelle Validität) zu ermitteln.

Die Validierung und Normierung des Modells und Instruments wurden im Rahmen verschiedener Veröffentlichungen bereits publiziert (vgl. Dörr et al. 2017b; SchmidtHuber et al. 2014; Schmidt-Huber 2011) und es liegt ein standardisierter, veröffentlichter LEaD-Fragebogen als Testverfahren vor (vgl. Dörr et al. 2017a). Vor diesem Hintergrund wird im Folgenden auf die zentralen Ergeb- 
Tab. 1 Auf einen Blick: ausgewählte empirische Ergebnisse der Validierungsstudie (Schmidt-Huber et al. 2014)

\section{Inhaltsvalidität \& Reliabilität}

Itemstatistik: Die Kennwerte der Items sprechen mit Mittelwerten von $2,88<M<4,15$ und Standardabweichungen von $0,92<S D<1,27$ für die Differenzierungsfähigkeit des Instruments, die Trennschärfen der Items $\left(r_{i t}>0,54\right)$ verweisen auf die Prototypizität der Items für die konstruierten Dimensionen (Bühner 2006), die Faktorladungen $(\lambda>0,57)$ und Kommunalitäten $\left(R^{2}>0,32\right)$ legen zudem eine gute inhaltliche Erfassung und Homogenität der LEaD-Kompetenzen (=Dimensionen) nahe. Mithilfe der Validierungsstudie konnte der Itempool durch Selektion der psychometrisch besten Items weiter auf 54 Items reduziert werden.

Die Ergebnisse der Validierung verweisen auf gute Reliabilitätswerte (Cronbachs Alpha für Faktoren-Ebene: 0,82< $\alpha<0,92$; Cronbachs Alpha für Dimensionen-Ebene: $0,79<\alpha<0,91)$.

Die inhaltliche Validität wurde durch die iterative Entwicklungsstrategie sichergestellt: Für jede LEaD-Kompetenz wurden verhaltensnahe Fragen aus dem theoretischen Fundus und Behavioral-Event-Interviews mit Führungskräften operationalisiert.

\section{Konstruktvalidität (faktorielle Validität, konvergente \& divergente Validität)}

Die fünffaktorielle Struktur des LEaD-Kompetenzmodells wurde empirisch im Rahmen der Schmid-Leimann-Prozedur (Verfahren zur Analyse der Interitemkorrelationen) sowie durch konfirmatorische Faktorenanalysen bestätigt (RMSEA =0,046, CFI =0,93, TLI=0,92 und NFI =0,90). Die Interkorrelationen der Kompetenzen liegen mit $r=0,18$ bis $r=0,75(p<0,001)$ in einem akzeptablen Bereich, sodass die Existenz von Konstruktredundanzen ausgeschlossen werden kann (Bühner 2006). Im Durchschnitt teilen die Kompetenzen 37,9\% der Konstruktvarianz.

Die LEaD-Kompetenzen wurden weiterhin von den Teilnehmenden von ineffektiven Verhaltensweisen ihrer Führungskräfte klar abgegrenzt, erkennbar in signifikanten Korrelationen mit den Skalen Laissez. Faire und Management by Exception passive des Multifactor Leadership Questionnaire (MLQ, Felfe und Goihl 2002; $r=-0,47$ bis $-0,71$ ).

Die substantiell höheren Zusammenhänge der Kompetenzen mit inhaltsnahen Skalen von Messinstrumenten mit einer ähnlichen Zielsetzung - Transformational Leadership Inventory (TLI) und Managerial Practices Survey (MPS), (Felfe und Goihl 2002; Podsakoff et al. 1990; Kim und Yukl 1995) - belegen die Konstruktvalidität des Modells und Fragebogens ( $r=-0,46$ bis 0,68).

Gleichzeitig verweist die Höhe der geteilten Konstruktvarianzen mit publizierten Instrumenten darauf, dass mit LEaD keine Replikation bestehender Instrumente vorliegt, sondern ein eigenständiges Modell und Instrument entwickelt wurde: Die geteilte Varianz mit dem TLI beträgt $40,6 \%$, mit dem MPS $32,1 \%$.

\section{Kriteriumsvalidität}

Für die Kriteriumsvalidität des LEaD-Fragebogens sprechen die substantiellen Zusammenhänge der einzelnen LEaD-Dimensionen mit verschiedenen subjektiven motivationalen Erfolgsvariablen (Extra-Anstrengung $\left(R^{2}=0,61\right)$, Zufriedenheit mit der Führungskraft $\left(R^{2}=0,79\right)$, Effektivität der Führungskraft $\left(R^{2}=0,81\right)$, affektives Commitment $\left(R^{2}=0,40\right)$ ), subjektiven leistungsbezogenen Erfolgskriterien (Gruppenleistung $\left(R^{2}=0,27\right)$, Innovationsverhalten $\left(R^{2}=0,19\right)$, individuelle Leistung $\left(R^{2}=0,07\right)$ ) sowie dem zunehmend bedeutsameren Kriterium des Stresserlebens der Mitarbeiter $\left(R^{2}=0,25\right)$ sowie. Auch mit objektiven Erfolgsvariablen, wie z. B. 1-Item-Messung zu Fehlzeiten $\left(R^{2}=0,05\right)$ und zur variablen Vergütung $\left(R^{2}=0,16\right)$, resultieren teilweise signifikante Zusammenhänge.

Mit Ausnahme einer LEaD-Kompetenz („Probleme analysieren“) erweisen sich alle anderen LEaD-Kompetenzen im Rahmen von schrittweisen Regressionen bei mindestens einem Erfolgsmaß als statistisch bedeutsame Prädiktoren: Die höchste Erfolgsrelevanz weisen dabei die Kompetenzen „Zukunftsperspektive vermitteln“ (Strategieorientierung), „Verantwortung delegieren“, „Feedback geben“, „Perspektiven übernehmen“ (Mitarbeiter-/Teamentwicklung), ,Ressourcen bereitstellen“ (Umfeldgestaltung) und „Authentizität vermitteln“ (persönliche Einflussnahme) auf. Die zunehmende Bedeutung veränderungsorientierter Einflussstrategien zeigt sich vor allem darin, dass die LEaD-Kompetenzen ,Zukunftsperspektiven aufzeigen“, „Innovationen treiben“ und „Veränderungen managen“ signifikante Prädiktoren von Innovationsleistung und auch affektivem Commitment, emotionaler Bindung zum Unternehmen, darstellen.

Es konnten keine systematischen Unterschiede in den Zusammenhängen zwischen den LEaD-Dimensionen und den Erfolgsmaßen zwischen unterschiedlichen Hierarchieebenen ermittelt werden.

Die LEaD-Kompetenzen klären im Vergleich zu anderen bewährten Instrumenten zur Messung von Führungsverhalten (TLI und MPS) inkrementelle Varianzanteile zwischen 1 bis $20 \%$ auf, d. h. liefern hinsichtlich verschiedener Erfolgsmaße der Führung einen höheren Prognosewert.

Die Interraterübereinstimmungen zwischen den Urteilen der Mitarbeitenden je einer Führungskraft $(N=184,21$ Teams $)$ beträgt $r_{\mathrm{wg}}(3)=0,76$ bis 0,92 , die Intra-Klassenkorrelationswerte liegen bei einem durchschnittlichen ICC(1) von 0,31 sowie einem durchschnittlichen ICC(2) von 0,74. Die Ergebnisse weisen auf eine hohe Übereinstimmung der Mitarbeiterurteile und eine ausreichende Differenzierung der Kompetenzen zwischen den Teams hin. Der Kennwert ICC(1) drückt aus, mit welcher Genauigkeit im Durchschnitt ein*e einzelne*r Mitarbeiter*in das Führungsverhalten beurteilt. Der Wert ICC(2) repräsentiert die Schätzung der Reliabilität der Gruppendurchschnitte. In der Regel fällt ICC(2) höher aus als ICC(1), da gemittelte Werte mehrerer Beurteiler*innen individuelle Fehleranteile ausgleichen können (vgl. Bliese 2000).

Die Validierungsstichprobe $(N=765)$ setzt sich aus Teilnehmenden unterschiedlichster Branchen (v. a. Finanzdienstleistung, Gesundheits- und Sozialwesen, Industrie, Information \& Kommunikation, Dienstleistung), Hierarchieebenen (22,2\% unterstes, $43,1 \%$ mittleres, $34,7 \%$ oberstes Management) und Tätigkeitsfeldern zusammen, die jeweils die LEaD-Kompetenzen ihrer aktuellen oder ehemaligen Führungskraft einschätzen (Online-Fragebogen, Dauer ca. $30 \mathrm{~min}, 227$ Items) 
Tab. 2 Ökonomie, Handhabbarkeit \& Akzeptanz des LEaD-Modells

\section{Ökonomie \& Handhabbarkeit}

Das Kompetenzmodell umfasst 5 Kompetenzbereiche und 18 Kompetenzen, die mit einem Fragebogen (,LEaD-Feedback“) mit 54 Items (3 Items je Kompetenz) erhoben werden können.

Die Befragungsdauer von ca. 15 bis 20 min für das LEaD-Feedback im Selbst- und Fremdbild ermöglicht ökonomische Befragungsprozesse und wird mit externer Unterstützung bedarfsorientiert gesteuert und begleitet.

Die Erhebungssoftware zum Online-Fragebogen kann flexibel genutzt werden und ermöglichen eine unkomplizierte Kommunikation mit den Teilnehmenden.

Die Inhalte können modular variiert werden und sichern damit die Anschlussfähigkeit an unternehmensspezifische Leitbilder und bestehende Kompetenzmodelle.

\section{Akzeptanz}

Evidenzbasierte und erfolgskritische Kompetenzbereiche des LEaDModells ermöglichen eine breite Anwendbarkeit und tragen zur Akzeptanz unter den Anwendern bei (hoher Grad an Plausibilität) darüber hinaus werden die Standards zur Umsetzung von Führungsfeedback berücksichtigt (vgl. London 2003):

Die Anonymität für Feedbackgebende schafft die Basis für offene Rückmeldungen.

Durch die externe Steuerung des Befragungsprozesses sind die Rückmeldungen vertraulich und nicht verfälschbar.

Die gewährleistete Prozesstransparenz für alle Beteiligten gibt Sicherheit zum Umgang mit den Daten, den Ergebnissen und Rückmeldeprozessen.

Feedback zum Ergebnisbericht unterstützt den konstruktiven Umgang mit den Ergebnissen und fördert die Nachhaltigkeit der Umsetzung.

Beratung zu Einbindung der Feedbackgebenden in die Ergebnisrückmeldung unterstützt Informationstransparenz, fördert Kooperationsprozesse und sorgt für einen verantwortungsvollen Umgang mit den Befragungsergebnissen.

nisse der Validierung zur Überprüfung der Gütekriterien eingegangen (Tab. 1).

Das evidenzbasierte LEaD-Modell und der LEaD-Fragebogen erfüllen wesentliche wissenschaftliche Anforderungen (z.B. Gütekriterien der Reliabilität und Validität sowie Orientierung an Standards für psychologisches Testen) und praktische Anforderungen (z.B. Ökonomie, Akzeptanz, Adaptabilität an Anforderungen, Aufzeigen von Ansatzpunkten für Interventionen, etc., vgl. z.B. Kauffeld und Paulsen 2018). Die 18 LEaD-Dimensionen wurden tätigkeits- und branchenübergreifend konzipiert und können für verschiedene Führungspositionen und Hierarchieebenen eingesetzt werden. Durch die theoretische Verankerung des Modells steht ein erprobtes Kompetenzmodell zur Verfügung, das sowohl evidenzbasierte als auch praxisrelevante Inhalte kondensiert integriert. Der Umfang des LEaD-Modells lässt dabei organisationsspezifische Erweiterungen zu, sodass spezielle Anforderungen, Kulturspezifika und Rahmenbedingungen von Organisationen oder Führungspositionen berücksichtigt werden können, wie z.B. die Begrifflichkeiten der Führungsleitsätze/des Führungsmodells einer
Organisation mit dem LEaD-Modell in Einklang zu bringen oder spezifische Kompetenzdimensionen des Unternehmens als Erweiterung zu LEaD aufzunehmen (Praxisbeispiel siehe Dörr et al. 2016).

In Praxiseinsätzen wird $\mathrm{LEaD}$ in enger Zusammenarbeit mit den Anwender*innen bzw. dem Unternehmen vor dem Hintergrund des jeweils verfolgten Umsetzungsziels gestaltet. Vor dem Einsatz erfolgt eine umfassende Situationsund Bedarfsanalyse, unabhängig vom Anwendungsfeld des Modells. Ein typischer Einsatzbereich ist das Führungsfeedback, das in 90-Grad bis 360-Grad-Varianten zur Verfügung steht und in Form eines Online-Befragungsprozesses gesteuert werden kann (vgl. Dörr et al. 2017a, b).

Im Rahmen der Anwendung von LEaD wird zudem der Anspruch verfolgt, auch den klassischen Anforderungen an Ökonomie, Handhabbarkeit und Akzeptanz von Testverfahren nachzukommen (vgl. Tab. 2). Der bereits mehrjährige Einsatz des LEaD-Modells hat gezeigt, dass Anwender*innen neben dessen inhaltlicher Ausgestaltung besonders auch den praxisorientierten Ansatz schätzen.

Abschließend gibt Abb. 1 einen Überblick über den gesamten Entwicklungsprozess des LEaD-Modells: Zunächst wurden verschiedene erfolgsversprechende Führungskonzepte integriert und im Rahmen wissenschaftlicher Studien auf deren Validität und Anwendungsflexibilität hin überprüft. Aus den Validierungsstudien wurden dann die Inhalte des LEaD-Modells schrittweise präzisiert und evidenzbasiert verdichtet. Die Anwendung in der Praxis erfolgt anforderungsbezogen und ermöglicht die Durchführung diagnostischer Maßnahmen, wie z.B. dem bereits beschriebenen Führungsfeedback oder einem Managementaudit sowie die inhaltliche Ausgestaltung von Entwicklungsinterventionen wie Coachingmaßnahmen, Trainingsformate und Beratung zu Change-Management.

\section{Veränderungen in der Arbeitswelt mit Auswirkungen auf die Führung}

Die Arbeitswelt wird, unabhängig von Branche und Organisationsziel, virtueller, flexibler und schneller (Maier et al., 2020). Dabei lassen Entwicklungen im Bereich der Robotik (Steil und Maier 2017, 2020), der Nutzung von AR-Brillen im betrieblichen Alltag (vgl. z. B. Bentler et al. 2019; Paruzel et al. 2020) oder der zunehmenden Digitalisierung des Auftragsdurchlaufs (Schlicher et al. im Druck) nur erahnen, wie die Arbeitswelt von morgen aussieht. Der Fokus über die Auswirkungen der „,neuen“ Technologien lag bislang vornehmlich auf den Mitarbeiter*innen (vgl. z.B. Ötting et al. 2020a) und (noch) nicht so sehr auf den Führungskräften.

Schon seit einiger Zeit kursieren Schätzungen darüber, wie viele Berufe in der Arbeitswelt der Digitalisierung zum 
Abb. 1 Entwicklung und Anwendung des LEaD-Kompetenzmodells im Überblick

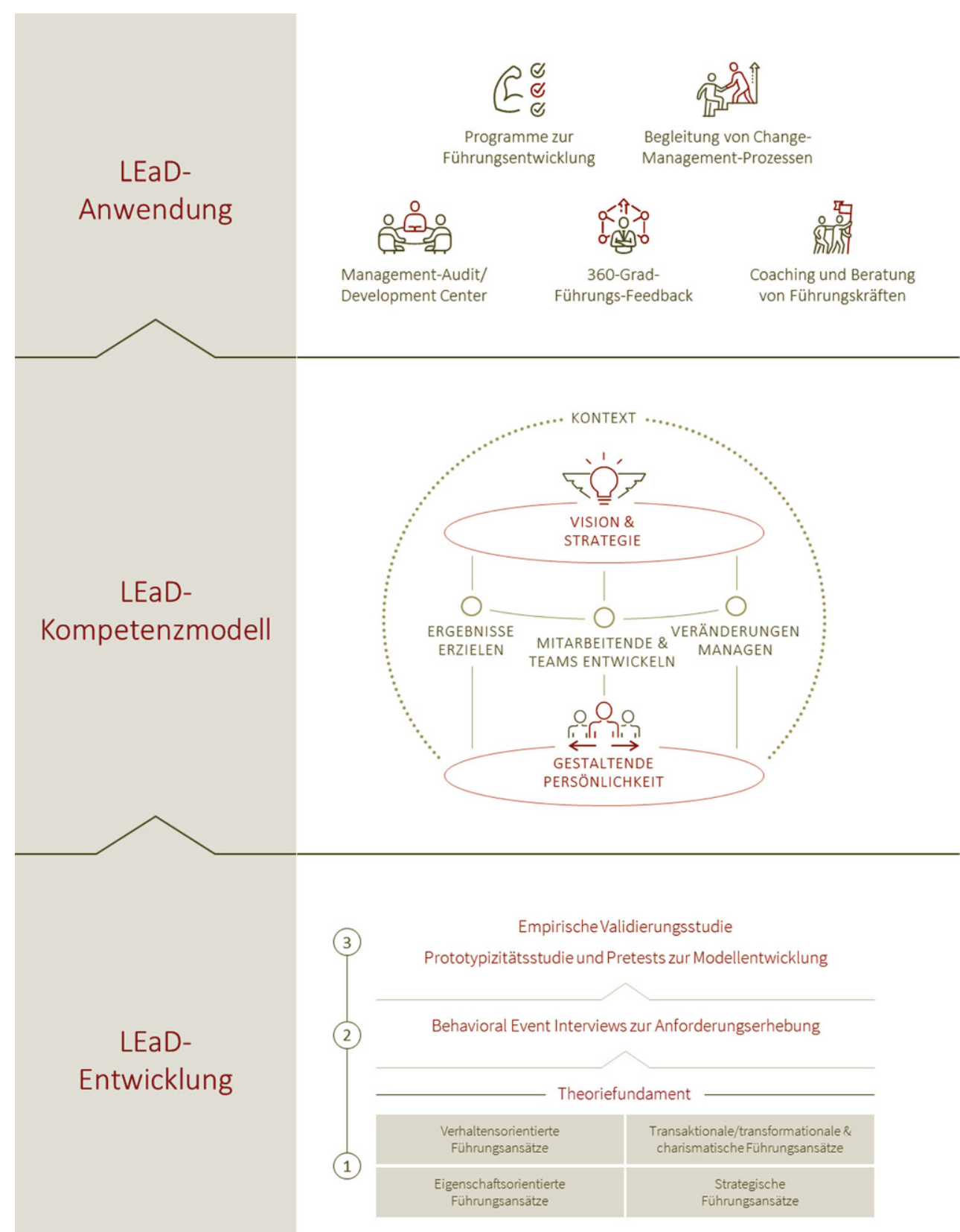

matisierungsmöglichkeit an (https://job-futuromat.iab.de/). Das Dilemma bei der zunehmenden Digitalisierung besteht darin, dass Beschäftigte inzwischen oftmals mit Angst auf diese sich abzeichnenden Veränderungen reagieren (Pfaffinger et al. 2020). Des Weiteren wird vielfach behauptet, dass Organisationen, die den Anschluss an den Trend der Digitalisierung versäumen, langsamer, unflexibler und unwissender als die digitalen Vorreiter seien und damit mittel- bis langfristig aus dem Markt verdrängt würden (vgl. O'Reilly und Tushman 2016).

Führungsaufgaben werden von der zunehmenden Digitalisierung nicht verschont bleiben. Betroffen sind die Führungsaufgaben dadurch, dass sie vollständig substiTeamleiter*innen zeigt beispielsweise eine 33\%ige Auto- 
tuiert werden, Führungskräfte durch digitale Technologie unterstützt werden (digitale Assistenten) oder sich neue Aufgabenfelder bzw. -inhalte abzeichnen. Ein an Bedeutung zunehmendes Aufgabenfeld ist dabei sicherlich, digitale Veränderungen im eigenen Umfeld zu bewältigen, entsprechende Technologie erfolgreich im eigenen Bereich einzuführen, dabei vorhandene Aufgabenfelder im Team neuzugestalten und vor allen Dingen die eigenen Teammitglieder bei der Einführung der neuen Technologie zu unterstützen und sie für die Nutzung der neuen Technologie zu gewinnen. Ebenfalls ein neues Feld dürfte es dabei auch sein, hybride Teams zu führen. Nicht nur technikorientierte Publikationen (vgl. z.B. Seeber et al. 2020), sondern mittlerweile auch in der Führungsforschung wird davon ausgegangen, dass es in Zukunft auch hybride Teams (zusammengesetzt aus Menschen und weitgehend gleichberechtigten technischen Agenten) geben wird (vgl. z.B. Larson und DeChurch 2020), die auch geführt werden müssen.

Digital vermittelt Führung über entsprechende Kommunikationsplattformen, wie z.B. e-leadership (Avolio et al. 2000), war in dieser Entwicklung lediglich ein erster Zwischenschritt. Bei diesen Formen digital vermittelter Führung hatte die Technologie keinen eigenständigen Beitrag bei der Erledigung von Führungsaufgaben (Wesche und Sonderegger 2019), sondern war lediglich ein Medi- um, über das Teams gebildet und geführt wurden (Larson und DeChurch 2020).

Schwarzmüller et al. (2015) haben in einer Studie zu den Herausforderungen für Organisationen in der digitalisierten Welt, wie z. B. zunehmende Flexibilisierung, Demokratisierung und Konnektivität, entsprechende neue Anforderungen an Führungskräfte abgeleitet (vgl. Welpe et al. 2018). Beispielsweise werden Regelungen der Zusammenarbeit und Delegation von Arbeitsaufgaben an Bedeutung gewinnen, damit ein eigenverantwortliches Handeln und effektives Arbeiten gewährleistet wird. In Interviews mit Führungskräften und Expert*innen fanden Schwarzmüller et al. (2016) drei besondere Schwerpunkte, wie sich das „Mindset“ und das Verhalten effektiver Führung in Zukunft verändern müssen:

1. Zuallererst sind von Führungskräften veränderte Einflussstrategien gefordert: Sie müssen mehr Macht abgeben, Autonomie fördern und eine Zusammenarbeitskultur von Vertrauen und Partizipation stärken.

2. Weiterhin rückt beziehungsförderliches Verhalten stärker in den Fokus, was z. B. bei agiler Team- und Projektarbeit eine zentrale Bedeutung spielt: Führungskräfte müssen stärker auf individuelle Bedürfnisse eingehen und Wertschätzung für Personen, deren Expertise, Arbeitsstile und Kompetenzen vermitteln. Eine übergreifende $\mathrm{Zu}$ -

Tab. 3 Exemplarische marktbezogene und technische Entwicklungen in übergeordneten Kompetenzfeldern von Führungskräften

Übergeordnete Kompetenzfelder im LEaD- Marktbezogene und technologische Entwicklungen

Modell

Strategieorientierung: $\quad$ Roboter als Vorstandsmitglied: Zolfagharifard (2014)

Chancen erkennen, Zukunftsperspektiven

formulieren, Innovationen fördern

Ergebnissicherung:

Ziele vereinbaren, Probleme analysieren, Ergebnisse bewerten

Mitarbeiter-/Teamentwicklung:

Verantwortung delegieren, Feedback geben,

Mitarbeiter coachen, Perspektiven übernehmen

Umfeldgestaltung:

Effektiv kommunizieren, Ressourcen

bereitstellen, Konflikte managen,

Veränderungen umsetzen, Beziehungen

managen

Persönliche Einflussnahme:

Selbstvertrauen vermitteln, Authentizität aus-

strahlen, Ambiguitäten managen
Roboter als Vorstandsmitglied: Zolfagharifard (2014)
Großer Anteil der Tätigkeiten von Non-Executive Directors kann durch KI unterstützt oder ersetzt werden: Jesuthasan und Ganu (2019)

Diskussion von Vor- und Nachteilen der Integration von KI in den Vorstand: Hilb (2020)

Neue Geschäftsmodelle durch Ambidextrie: O'Reilly und Tushman (2016)

Algorithmisches, datengetriebenes Management (Ziele vorgeben, Leistung bewerten): Lee et al. (2015)

Roboter geben Ziele vor und ermuntern zur Einhaltung: Geiskkovitch et al. (2015)

Roboter weisen Aufgaben zu: Gombolay et al. (2014)

Aufgabenverteilung und Entscheidung über Trainings (Gedankenexperimente): Ötting und Maier (2018, 2021)

Entscheidung über Urlaub: Schlicker et al. (2021)

Einsatz von Augmented Reality (AR) statt erfahrener Person (z. B. Meister, Ausbilder, Schichtleiter) zum Anlernen von Beschäftigten: z. B. Neumann et al. (2020); Ometov et al. (2021); Palmarini et al. (2018)

KI unterstütztes virtuelles Bewerbertraining, inkl. Feedback: Langer et al. (2016)

Teamzusammensetzung Algorithmus-gesteuert unterstützen: MyDreamTeam (s. Larson und DeChurch 2020)

Personalauswahl unterstützt durch KI: z. B. Langer et al. (2020a, b)

Führungskräfte müssen in Zukunft verstärkt den Wandel gestalten: z. B. Schein und von Ameln (2019); Ötting et al. (2020b)

„Agiles Mindset“ der Führung durch mehr Zusammenarbeitskultur, beziehungsförderliches Verhalten und agiles Management notwendig: Welpe et al. (2018) 
sammenarbeit - weg von „Silos“ - erfordert ein stärkeres Teambuilding.

3. Schließlich kommt Führungskräften eine immer stärkere Rolle als „Enabler“ zu, um erhöhte Kompetenzanforderungen zu managen: Dabei führen sie Mitarbeitende und Teams stärker auf Distanz, bündeln die Leistungen zunehmend in diversen Teams.

Die in diesem Zusammenhang wachsende Bedeutung der geteilten Führung stellt keinen Gegensatz zu klassischer Führung durch eine Person dar. Empirisch konnte gezeigt werden, dass vertikale Führung den Einfluss geteilter Führung sogar verstärken kann (Grille et al. 2015).

Weitere Kompetenzanforderungen entstehen inhaltlich durch neue Geschäfts- und Vertriebsmodelle und technologisch durch neue Methoden- und IT-Anforderungen. Führungskräfte müssen in der Lage sein, neben dem Kerngeschäft neue innovative Geschäftsmodelle zu entdecken und zu entwickeln. O'Reilly und Tushman (2016) haben in dem Ansatz der Ambidextrie die Herausforderung an Führung beschrieben, die zunächst ambivalenten Fähigkeiten „to exploit“ (Kerngeschäft der Organisation zu managen) und „to explore“ (Innovationsleistungen zu fördern) gleichzeitig managen zu können. Unternehmen, so die Autoren, die effizient und ökonomisch wirtschaften, sind nicht zwangsläufig auch in der Lage, neue innovative Geschäftsfelder zu entwickeln und zu etablieren. Im Gegenteil: Eine hohe ökonomische Effizienz kann sich sogar als hinderlich oder unvereinbar mit einer Innovationskultur erweisen. O'Reilly und Tushman (2016) heben vier Erfolgsfaktoren hervor, wie Ambidextrie im Unternehmen umgesetzt werden kann. Zunächst bedarf es 1) einer klaren strategischen Ausrichtung, die sich auf eine Entwicklung innovativer Geschäftsfelder fokussiert, neue Märkte erschließt und neue Kompetenzen hierfür aufbaut. Darüber hinaus erfordert Ambidextrie 2) ein klares Commitment des Top Managements hinsichtlich finanzieller, organisatorischer und personeller Anforderungen. Es bedarf 3) einer strukturellen Architektur in Unternehmen, die es möglich macht, beide Geschäftsstrategien (to exploit und to explore) simultan umzusetzen, jedoch in unabhängigen organisatorischen Einheiten. Die gemeinsame Identität für das Unternehmen wird durch 4) eine übergreifende Vision und Kultur geschaffen. In vielen Projekten hat sich gezeigt, dass neben einer einheitlich kommunizierten Vision, Strategie und übergreifenden Werten auch eine ausgeprägte Konfliktfähigkeit vom gesamten Management gefordert ist.

Im Folgenden soll kurz darstellt werden, welche Kompetenzfelder klassischer Führungskräfte durch aktuelle marktbezogene und technische Entwicklungen der Digitalisierung tangiert werden, indem exemplarisch gezeigt wird, welche digitalen Entwicklungen sich aktuell in diesen klassischen Feldern abzeichnen (vgl. Tab. 3). Grundsätzlich ist es kein völlig neues Phänomen, dass die Einführung neuer Technologien bestehende Rollen in Organisationen in Frage stellt und sie teilweise neu ausgehandelt werden müssen (vgl. z.B. eine Studie zur Einführung von CT-Geräten in Radiologischen Abteilungen von Krankenhäusern: Barley 1986). Neu an den aktuellen Technologien ist, dass sie sich mit (z. B. Roboter) oder ohne (z. B. KI, Bots) Körperlichkeit sehr flexibel an neue Situationen anzupassen beginnen.

Was zeigt die exemplarische Übersicht in Tab. 3? Erstens wird deutlich, dass alle Führungsebenen, begonnen mit Team- und Schichtleiter*innen bis hin zum Top-Management und der Geschäftsführungsebene von diesen Entwicklungen betroffen sind. Zweitens zeigt sich, dass viele Kompetenzfelder betroffen sind: Insofern als technische Agenten schon Vorstandsmitglieder sind, ist die Strategieorientierung betroffen. Ziele vereinbaren, Aufgaben verteilen und der Personaleinsatz wird in mehr oder weniger großem Umfang KI-unterstützt gesehen und die Auswirkungen analysiert - insofern ist die Ergebnissicherung betroffen. Auch im Bereich der Mitarbeiterentwicklung kommen neue Technologien zum Einsatz, z.B. beim Anlernen von Beschäftigten in der Produktion durch KI-gestützte AR-Technologie. Neben der verstärkten Kompetenzförderung müssen Führungskräfte stärker Autonomie fördern und mit agilen Methoden die übergreifende Zusammenarbeit fördern. Auch bei der Gestaltung des Umfelds kommt KI zum Einsatz, z.B. für die Zusammenstellung optimaler Teams, oder die Personalauswahl wird durch KI unterstützt und die Folgen davon untersucht. Drittens zeigt sich hinsichtlich der Persönlichen Einflussnahme, dass einerseits eine neues „Mindset" der Führung gefragt ist, aber andererseits noch kaum technologische Entwicklungen ausgemacht werden konnten, die diesen Kompetenzbereich betreffen.

\section{Implikationen für die Praxis}

Der Kontext der digitalen Transformation erfordert eine Führungskultur, in der Führungskräfte verstärkt den Wandel gestalten (Ötting et al. 2020b), wie eine Studie mit Expert*innen aus KMU zeigt. In dieser Rolle als „Change Leader" (vgl. Bohn et al. 2015) und Gestalter*innen sind sie verstärkt gefordert, „Brücken zu bauen, übergreifende $\mathrm{Zu}$ sammenarbeit herzustellen, Mitarbeitende zu befähigen und zu inspirieren" (vgl. Preusser und Bruch 2014). Das bedeutet, dass es neben der Berücksichtigung der sich vermehrt ändernden Kompetenzen der Beschäftigten (vgl. z. T. Schlicher et al. im Druck) genauso wichtig sein wird, auch die sich daraus ändernden Kompetenzen von Führungskräften im Fokus zu behalten und nicht zu vernachlässigen. Dabei ist besonders darauf zu achten, dass nicht nur die aktuellen, sondern auch die zukünftigen Kompetenzen der Führung ermittelt werden (vgl. Kato-Beiderwieden et al. im Druck). 
Abb. 2 „Beidhändige Führung“ mit dem LEaD-Modell im Spannungsfeld von Traditioneller Führung und Digital Leadership
TRADITIONELLE FÜHRUNG

DIGITAL LEADERSHIP

\begin{tabular}{|c|c|c|}
\hline Kerngeschäft vorantreiben & < Strategieentwicklung> & Innovation fördern \\
\hline ("exploit") & Marktchancen erkennen, Zukunftsperspektiven formulieren & ("explore") \\
\hline Top-Down Prozesse & & Bottom-up Prozesse \\
\hline
\end{tabular}

Im Fokus sind lineare Planungsprozesse und kaskadenförmige Umsetzungsprozesse, zentrale Anweisungen und Entscheidungen liegen im Management - das Management hat Definitionsmacht

\begin{tabular}{|c|c|c|}
\hline Vorgabe und Kontrolle & $<$ Ergebniserreichung > & Selbstverantwortung stärken \\
\hline Einzelziele / -leistung & Ziele vereinbaren, Probleme analysieren, Ergebnisse bewerten & Teamziele / -leistung \\
\hline
\end{tabular}

Zentral sind klare Ziel- und Jahresplanungen in festge- Es kommt vor allem auf flexible Planungsprozesse an: legten Beurteilungszeiträumen: Führungskräfte steuern, Führungskräfte fördern Selbststeuerung, kontinuierliplanen und kontrollieren Ergebnisse, sie vermeiden che Reflexion der Qualität und Ergebnisse und lernen Fehler aus Fehlern

$\left.\begin{array}{l}\text { Management (transaktional) } \\ \text { "Silodenken“ }\end{array} \quad \begin{array}{l}\text { < Mitarbeiter-/Teamentwicklung > } \\ \text { Verantwortung delegieren, Feedback geben, Mitarbeitende } \\ \text { coachen, Perspektiven übernehmen }\end{array}\right) \begin{array}{r}\text { Leadership (transformational) } \\ \text { übergreifende } \\ \text { Zusammenarbeit }\end{array}$

Führung geschieht in erster Linie durch Planung, Anwei- Führung meint in erster Linie Empowerment und sung und Kontrolle, Informationen fließen von oben Coaching, Verantwortung zu übertragen und offenes nach unten, Lernen erfolgt vorwiegend durch zentrale Feedback zu geben, Information und Wissen zu teilen, Weiterbildungsangebote; Leistung steht vor individuel- Selbstlernprozesse anzustoßen, Leistung und Achtlen Bedürfnissen samkeit für Gesundheit sowie Work-Life-Balance im Blick zu behalten

$\left.\begin{array}{l|c}\hline \text { Effizienz und Stabilität } & \multicolumn{1}{c}{<\text { Umfeldgestaltung> }} \\ \text { Problemorientierung } & \begin{array}{l}\text { Effektiv kommunizieren, Ressourcen bereitstellen, Konflikte } \\ \text { managen, Veränderungen umsetzen, Beziehungen managen }\end{array}\end{array}\right) \begin{array}{r}\text { Wachstum \& Veränderung } \\ \text { Lösungsorientierung }\end{array}$

Im Vordergrund steht Risikominimierung durch hohen Innovation entsteht durch Experimentieren und NutKostendruck, wenig Spielraum für Kreativität und Inno- zen von Opportunitäten, der Anwendung agiler Mevation durch formale Kommunikationswege und Kon- thoden wie z.B. Scrum und einer konstruktiven Fehlerfliktvermeidung - die Führungskraft arbeitet „im Sys- lernkultur - die Führungskraft arbeitet „am System“ tem" (Change Controller) (Change Leader), macht Erfolge sichtbar und betrachtet Konflikte als Chance

\begin{tabular}{l|l}
\hline Hierarchie- & \multicolumn{1}{c}{$<$ Persönliche Einflussnahme > } \\
und Positionsmacht & $\begin{array}{l}\text { Selbstvertrauen vermitteln, Authentizität ausstrahlen, Ambigui- } \\
\text { tät managen }\end{array}$
\end{tabular}

Formelle Macht und hierarchische Entscheidungen ste- Transparente und verbindliche Werte und Rollen stärhen im Vordergrund mit der Gefahr, Intransparenz und ken eine Vertrauenskultur durch Beteiligung, dynamiAbgrenzungen zu erzeugen - der individuelle Machter- sche Entscheidungen, die Mut zum Risiko ermöglichen halt und die Angst vor Konsequenzen führen dazu, dass - Führungskräfte handeln authentisch, empathisch Führungskräfte oftmals Risiko, Unsicherheit und Ambi- und sind in der Lage, Zielkonflikte zu managen valenz vermeiden
Mit dem LEaD-Modell steht dafür ein unternehmensunabhängiges Kompetenzmodell zur Verfügung, das auch die zukünftigen Kompetenzen berücksichtigt und abbildet.

In Abb. 2 werden die LEaD-Kompetenzen im Sinne einer ,,beidhändigen Führung“ im Spannungsfeld von traditioneller Führung und Führung in digitalem Wandel (Digital Leadership) aufgezeigt. Dieses Spannungsfeld ist für Führungskräfte ein Balanceakt, der sie vor die Herausforderung stellt, zum einen das aktuelle Kerngeschäft effektiv zu managen und zum anderen flexibel und innovativ neue Geschäftsmodelle zu entwickeln. Diese Führungskomplexität wird auch mit ,,beidhändiger Führung“ umschrieben (Dörr et al. 2018; Petry 2016). Im Vordergrund steht dabei immer ein situationsgerechtes Ausbalancieren des individuellen Führungsverhaltens (vgl. Grote et al. 2009), aus- gehend davon, ob eher ein traditionelles oder ein digitales Führungsverhalten situativ erforderlich und zielführend ist.

Die aktuell im Rahmen der digitalen Transformation als digital Leadership bezeichneten Führungsansätze (vgl. Buhse 2012, 2014; Dörr et al. 2018) favorisieren ein Führungsverhalten, das stärker dem New Leadership Approach entspricht. Das Konzept der Ambidextrie oder beidhändigen Führung (O'Reilly und Tushman 2016) steht dabei für ein „sowohl als auch-Prinzip“ der Führung, bei dem ein aufgaben- und ergebnisorientiertes mit einem visionärbeziehungsorientiertem Führungsverhalten ausbalanciert wird, um die ökonomische Effizienz und innovative Transformation im Unternehmen zu erreichen.

Wirksames Führungsverhalten kann mithilfe des LEaDModells in der Unternehmenspraxis im Sinne einer ,ent- 
wicklungsorientierten Diagnostik“ (vgl. Dörr et al. 2016) sowohl gemessen als auch entwickelt werden. Die Anwendung in der Praxis erfolgt dabei auf individueller und organisationaler Ebene:

Auf individueller Ebene als

- Feedbackinstrument zum Führungsverhalten mit bis zu 4 Fremdperspektiven $\left(90^{\circ}-/ 180^{\circ}-/ 270^{\circ}-/ 360^{\circ}\right.$-Feedback),

- Instrument zur Identifikation von Potenzialen und Entwicklungsbedarfen im Rahmen der Führungskräfteauswahl und -entwicklung, z. B. als Management-Audit,

- Informationsquelle bei Potenzialeinschätzungsverfahren zur Steigerung der prognostischen Validität und

- Instrument zur Veränderungsmessung und Erfolgskontrolle für effektive Führung.

\section{Auf organisationaler Ebene zur}

- Beschreibung von Soll-Anforderungen zu erwarteten Führungskompetenzen,

- inhaltlichen Gestaltung von Führungsentwicklungsprogrammen,

- Gestaltung von Change-Management-Prozessen,

- Evaluierung und Identifikation von Verbesserungspotenzialen der bestehenden Führungskultur von Unternehmen oder einzelnen Unternehmensbereichen sowie

- Erstellung unternehmens- und positionsübergreifender Benchmarks zu Führungskompetenzen.

Eine Erweiterung der Messung von Führungskompetenzen in der Praxis könnte künftig durch eine verstärkte Diagnostik implizierter Motive erzielt werden (vgl. Jacobs und McClelland 1994). Untersuchungen zum sog. „Leadership Motiv Pattern" zeigten, dass sich vor allem das sozialisierte Machtmotiv günstig auf den Führungserfolg auswirkt (vgl. McClelland und Boyatzis 1982). Jüngere Studien heben vor allem die Bedeutung des immer wichtiger werdenden Beziehungsmotivs (vgl. z.B. Steinmann et al. 2015, 2020, 2016) hervor, da Führungskräfte in Zeiten zunehmender Veränderungen stärker gefordert sind, vertrauensvolle Kooperationen aufzubauen. Insofern ist es sinnvoll, die Messung von Kompetenzen mittels des LEaD-Modells um die Diagnose impliziter Motive zu erweitern, wie erste Anwendungen in der Praxis zeigen (Dörr et al. 2016).

\section{Fazit}

Nach einer ersten ,wilden“ Anfangsphase mit der Entwicklung vieler unternehmensspezifischer Kompetenzmodelle beginnt seit einigen Jahren auch eine wissenschaftliche Aufarbeitung der zunächst aus der Praxis heraus entstandenen Kompetenzmodellierung. Die frühe Entwicklung wurde in Deutschland - vor allem aus akademischer Perspektive - zurückhaltend und kritisch mit der Frage verbunden, ob die bisherige Anforderungen ablösenden Kompetenzen nicht lediglich methodisch unzulänglich gekelterter ,alter Wein in neuen Schläuchen" sind (vgl. hier z.B. Ebner et al. 2018; Sarges 2006). Inzwischen sind neben praktischen Erfordernissen auch wissenschaftsnahe Kriterien bei der Entwicklung berücksichtigt worden, nach solchen Kriterien können Benchmarks durchgeführt werden (vgl. Liebenow et al. 2020) und wir glauben mit der Entwicklung des LEaD-Modells einen Beitrag dazu geleistet zu haben.

Zusammenfassend lässt sich festhalten, dass effektive Führung gerade im Kontext des digitalen Wandels ein Schlüsselfaktor für den Unternehmenserfolg bleibt. Ein „Return on Leadership“ wird jedoch nur erzielt werden, wenn effektive Führung als „harte Währung“ verstanden wird und Unternehmen in die professionelle Auswahl und Entwicklung ihrer Führungskräfte investieren (Avolio et al. 2010). Das evidenzbasierte LEaD-Modell bietet in diesem Zusammenhang ein praxistaugliches Instrumentarium für die Auswahl und Entwicklung von Führungskräften, die im Spannungsfeld von ökonomischer Effizienz und innovativer Transformation handeln.

Funding Open Access funding enabled and organized by Projekt DEAL.

Open Access Dieser Artikel wird unter der Creative Commons Namensnennung 4.0 International Lizenz veröffentlicht, welche die Nutzung, Vervielfältigung, Bearbeitung, Verbreitung und Wiedergabe in jeglichem Medium und Format erlaubt, sofern Sie den/die ursprünglichen Autor(en) und die Quelle ordnungsgemäß nennen, einen Link zur Creative Commons Lizenz beifügen und angeben, ob Änderungen vorgenommen wurden.

Die in diesem Artikel enthaltenen Bilder und sonstiges Drittmaterial unterliegen ebenfalls der genannten Creative Commons Lizenz, sofern sich aus der Abbildungslegende nichts anderes ergibt. Sofern das betreffende Material nicht unter der genannten Creative Commons Lizenz steht und die betreffende Handlung nicht nach gesetzlichen Vorschriften erlaubt ist, ist für die oben aufgeführten Weiterverwendungen des Materials die Einwilligung des jeweiligen Rechteinhabers einzuholen.

Weitere Details zur Lizenz entnehmen Sie bitte der Lizenzinformation auf http://creativecommons.org/licenses/by/4.0/deed.de.

\section{Literatur}

Antonakis, J., \& House, R. J. (2004). On instrumental leadership: beyond transactions and transformations. Omaha: UNL Gallup Leadership Institute Summit.

Avolio, B. J., Kahai, S., \& Dodge, G.E. (2000). E-leadership: Implications for theory, research, and practice. Leadership Quarterly, 11(4), 615-668. https://doi.org/10.1016/S1048-9843(00)00062$\mathrm{X}$.

Avolio, B. J., Avey, J.B., \& Quisenberry, D. (2010). Estimating return on leadership development investment. Leadership Quarterly, 21(4), 633-644. https://doi.org/10.1016/j.leaqua.2010.06.006.

Barley, S.R. (1986). Technology as an occasion for structuring: Evidence from observations of CT scanners and the social order of radiology departments. Administrative Science Quarterly, 31(1), 78-108. https://doi.org/10.2307/2392767. 
Bentler, D., Mlekus, L., Paruzel, A., Bansmann, M., Foullois, M., Jenderny, S., Woeste, L., Dumitrescu, R., Röcker, C., \& Maier, G. W. (2019). Einführung von Augmented Reality in der Produktentstehung. Technische Realisierung und Change-Management als Erfolgsfaktor für den Veränderungsprozess. In Gesellschaft für Arbeitswissenschaft e. V. (Hrsg.), Arbeit interdisziplinär analysieren - bewerten - gestalten. Dortmund: GfA-Press.

Bliese, P. D. (2000). Within-group agreement, non-independence, and reliability: Implications for data aggregation. In K.J. Klein \& S. W. J. Kozlowski (Hrsg.), Multilevel theory, research, and methods in organizations: Foundations, extensions, and new directions (S. 349-381). San Francisco: Jossey-Bass.

Boal, K. B., \& Hooijberg, R. (2001). Strategic leadership research: moving on. Leadership Quarterly, 11(4), 515-549. https://doi.org/ 10.1016/S1048-9843(00)00057-6.

Bohn, U., Crummenerl, C., \& Graeber, F. (2015). Superkräfte oder Superteam? Wie Führungskräfte ihre Welt wirklich verändern können. Change Management Studie 2015. Capgemini Consulting. https://www.capgemini.com/consulting-de/wp-content/ uploads/sites/32/2017/08/change-management-studie-2015_5 pdf. Zugegriffen: 20.05.2021

Borman, W.C., \& Brush, D. H. (1993). More progress toward a taxonomy of managerial performance requirements. Human Performance, 6(1), 1-21. https://doi.org/10.1207/s15327043hup0601_ 1.

Braun, S., Frey, D., Nübold, A., \& Maier, G. W. (2017). Führung. In H.-W. Bierhoff \& D. Frey (Hrsg.), Enzyklopädie der Psychologie (Sozialpsychologie: Vol. 3. Kommunikation, Interaktion und soziale Gruppenprozesse) (S. 543-598). Göttingen: Hogrefe.

Bühner, M. (2006). Einführung in die Test- und Fragebogenkonstruktion (2. Aufl.). München: Pearson.

Buhse, W. (2012). Changing the Mindset: Die Bedeutung des Digital Leadership für die Enterprise 2.0-Strategieentwicklung. In G. Lembke \& N. Soyez (Hrsg.), Digitale Medien im Unternehmen (S. 237-252). Berlin: Springer.

Buhse, W. (2014). Management By Internet: Neue Führungsmodelle für Unternehmen in Zeiten der digitalen Transformation. Plassen: Kulmbach.

Campion, M. A., Fink, A. A., Ruggeberg, B. J., Carr, L., Phillips, G. M., \& Odman, R.B. (2011). Doing competencies well: best practices in competency modeling. Personnel Psychology, 64(1), 225-262. https://doi.org/10.1111/j.1744-6570.2010.01207.x.

Diewald, M., Andernach, B., \& Kunze, E. (2020). Entwicklung der Beschäftigungsstruktur durch Digitalisierung von Arbeit. In G. W. Maier, G. Engels \& E. Steffen (Hrsg.), Handbuch Gestaltung digitaler und vernetzter Arbeitswelten (S. 435-451). Berlin: Springer.

Dörr, S. (2008). Motive, Einflussstrategien und transformationale Führung als Faktoren effektiver Führung. München: Rainer Hampp.

Dörr, S., Schmidt-Huber, M., \& Maier, G. W. (2012). LEaD. Entwicklung eines evidenzbasierten Kompetenzmodells erfolgreicher Führung. In S. Grote (Hrsg.), Die Zukunft der Führung (S. 415-435). Berlin: Springer.

Dörr, S. L., Schmidt-Huber, M., Inderst, F.X., \& Maier, G. W. (2016). Führungskompetenzen diagnostizieren und entwickeln: Anwendung des LEaD-Kompetenzmodells effektiver Führung in der Praxis. In J. Felfe \& R. van Dick (Hrsg.), Springer Reference Psychologie. Handbuch Mitarbeiterführung. Wirtschaftspsychologisches Praxiswissen für Fach- und Führungskräfte (S. 111-128). Berlin: Springer.

Dörr, S. L., Schmidt-Huber, M., \& Maier, G. W. (2017a). LEaD-Führungsfeedback: Fragebogen für Gruppen- oder Einzeltest für Einsatz bei Führungskräften und deren Mitarbeitern. Göttingen: Hogrefe.

Dörr, S.L., Schmidt-Huber, M., \& Maier, G.W. (2017b). Messung von Führungskompetenzen - Leadership Effectiveness and Development (LEaD). In J. Erpenbeck, L. v. Rosenstiel, S. Grote
\& W. Sauter (Hrsg.), Handbuch Kompetenzmessung (3. Aufl. S. 113-135). Stuttgart: Schäfer-Poeschel.

Dörr, S.L., Albo, P., \& Monastiridis, B. (2018). Digital Leadership Erfolgreich führen in der digitalen Welt. In S. Grote \& R. Goyk (Hrsg.), Führungsinstrumente aus dem Silicon Valley (S. 37-61). Berlin: Springer.

Ebner, K., Greif, S., \& Kauffeld, S. (2018). Humanressourcen und Personalentwicklung. In S. Greif \& K.-H. Hamborg (Hrsg.), Methoden der Arbeits-, Organisations- und Wirtschaftspsychologie. Enzyklopädie der Psychologie, Themenbereich B, Methodologie und Methoden, Serie III, Psychologische Interventionsmethoden, (Bd. 3, S. 145-213). Göttingen: Hogrefe.

Felfe, J. (2015). Trends der psychologischen Führungsforschung. Göttingen: Hogrefe.

Felfe, J., \& Goihl, K. (2002). Deutsche überarbeitete und ergänzte Version des Multifactor Leadership Questionnaire (MLQ). ZUMA-Informationssystem. Elektronisches Handbuch sozialwissenschaftlicher Erhebungsinstrumente. Version 5.

Fisseni, H.-J. (2004). Lehrbuch der psychologischen Diagnostik. Göttingen: Hogrefe.

Fleishman, E. A., Mumford, M. D., Zaccaro, S. J., Levin, K. Y., Korotkin, A. L., \& Hein, M. B. (1991). Taxonomic efforts in the description of leader behavior: A synthesis and functional interpretation. Leadership Quarterly, 2(4), 245-287. https://doi.org/10.1016/ 1048-9843(91)90016-U.

Frey, C. B., \& Osborne, M. A. (2017). The future of employment: How susceptible are jobs to computerisation? Technological Forecasting and Social Change, 114, 254-280. https://doi.org/10.1016/j. techfore.2016.08.019.

Geiskkovitch, D., Cormier, D., Seo, S., \& Young, J. (2015). Please continue, we need more data: an exploration of obedience to robots. Journal of Human-Robot Interaction, 5(1), 82-99.

Gombolay, M.C., Gutierrez, R. A., Sturla, G. F., \& Shah, J. A. (2014). Decision-making authority, team efficiency and human worker satisfaction in mixed human-robot teams. Proceedings of the Robots: Science and Systems (RSS). http://interactive.mit.edu/sites/ default/files/documents/Gombolay_RSS_2014.pdf. Zugegriffen: 20.05.2021

Grille, A., Schulte, E.-M., \& Kauffeld, S. (2015). Promoting shared leadership - A multilevel analysis investigating the role of prototypical team leader behavior, psychological empowerment and fair rewards. Journal of Leadership and Organizational Studies, 22(3), 324-339. https://doi.org/10.1177/1548051815570039.

Grote, S., Kauffeld, S., \& Weide, C. (2009). Das Balance Inventar der Führung. In S. Kauffeld, S. Grote \& E. Frieling (Hrsg.), Handbuch Kompetenzentwicklung (S. 107-123). Stuttgart: SchäfferPoeschel.

Hilb, M. (2020). Toward artificial governance? The role of artificial intelligence in shaping the future of corporate governance. Journal of Management and Governance, 24, 851-870. https://doi.org/10. 1007/s10997-020-09519-9.

Hiller, N. J., DeChurch, L. A., Murase, T., \& Doty, D. (2011). Searching for outcomes of leadership: a 25-year review. Journal of Management, 37(4), 1137-1177. https://doi.org/10.1177/ 0149206310393520.

Jacobs, R.L., \& McClelland, D.C. (1994). Moving up the corporate ladder: a longitudinal study of the leadership motive pattern and managerial success in women and men. Consulting Psychology Journal: Practice and Research, 46(1), 32-41. https://doi.org/10. 1037/1061-4087.46.1.32.

Jesuthasan, R., \& Ganu, S. (1. Okt. 2019) Can a board member's job be automated? Harvard Business Review. https://hbr.org/2019/10/ can-a-board-members-job-be-automated.

Kato-Beiderwieden, A.-L., Ötting, S. K., Schlicher, K. D., Heppner, H., \& Maier, G. W. (2021). Prospektive Kompetenzanalyse (ProKA) - Ein Verfahren zur Einschätzung von zukünftigen Kompetenzveränderungen. Gruppe. Interaktion. Organisation. Zeitschrift für 
Angewandte Organisationspsychologie (GIO). https://doi.org/10. 1007/s11612-021-00577-7. Online-Vorabpublikation.

Kauffeld, S., \& Maier, G. W. (2020). Digitalisierte Arbeitswelt. Gruppe. Interaktion. Organisation. Zeitschrift für Angewandte Organisationspsychologie (GIO), 51, 1-4. https://doi.org/10.1007/ s11612-020-00508-y.

Kauffeld, S., \& Paulsen, H. (2018). Kompetenzmanagement in Unternehmen. Kompetenzen beschreiben, messen, entwickeln und nutzen. Stuttgart: Kohlhammer.

Kim, H., \& Yukl, G. (1995). Relationships of managerial effectiveness and advancement to self-reported and subordinate-reported leadership behaviors from the multiple-linkage mode. Leadership Quarterly, 6(3), 361-377. https://doi.org/10.1016/10489843(95)90014-4.

Langer, M., König, C. J., Gebhard, P., \& André, E. (2016). Dear computer, teach me manners: testing virtual employment interview training. International Journal of Selection \& Assessment, 24(4), 312-323. https://doi.org/10.1111/ijsa.12150.

Langer, M., König, C. J., \& Busch, V. (2020a). Changing the means of managerial work: effects of automated decision support systems on personnel selection tasks. Journal of Business and Psychology. https://doi.org/10.1007/s10869-020-09711-6.

Langer, M., König, C. J., \& Hemsing, V. (2020b). Is anybody listening? The impact of automatically evaluated job interviews on impression management and applicant reactions. Journal of Managerial Psychology, 35(4), 271-284. https://doi.org/10.1108/JMP-032019-0156.

Larson, L., \& DeChurch, L.A. (2020). Leading teams in the digital age: four perspectives on technology and what they mean for leading teams. Leadership Quarterly, 31(1), 101377. https://doi.org/ 10.1016/j.leaqua.2019.101377.

Lee, M.K., Kusbit, D., Metsky, E., \& Dabbish, L. (2015). Working with machines: The impact of algrorithmic and data-driven management on human workers. In B. Begole, J. Kim, K. Inkpen \& W. Woo (Hrsg.), Proceedings of the 33rd annual ACM conference on human factors in computing systems (S. 1603-1612). : ACM. https://doi.org/10.1145/2702123.2702548.

Liebenow, D., Uedelhoven, S., Stricker, M., \& Nachtwei, J. (2020). Qualität im Kompetenzmanagement: Eine explorative Studie zur Entwicklung eines Benchmarks für Kompetenzmodellierung und Kompetenzmodell (BeKom). Zeitschrift für Arbeits- und Organisationspsychologie, 64(2), 120-148. https://doi.org/10.1026/ 0932-4089/a000324.

London, M. (2003). Job Feedback: Giving, seeking, and using feedback for performance improvement. Mahwah: Erlbaum.

Lord, R.G., Day, D. V., Zaccaro, S.J., Avolio, B.J., \& Eagly, A.H. (2017). Leadership in applied psychology: three waves of theory and research. Journal of Applied Psychology, 102(3), 434-451. https://doi.org/10.1037/ap10000089.

Maier G. W., Engels G., \& Steffen E. (Hrsg.) (2020). Handbuch Gestaltung digitaler und vernetzter Arbeitswelten. Berlin: Springer.

McClelland, D.C. (1998). Identifying competencies with behavioralevent interviews. Psychological Science, 9(5), 331-339. https:// doi.org/10.1111/1467-9280.00065.

McClelland, D.C., \& Boyatzis, R.E. (1982). Leadership motive pattern and long-term success in management. Journal of Applied Psychology, 67(6), 737-743. https://doi.org/10.1037/0021-9010. 67.6.737.

Neumann, A., Strenge, B., Uhlich, J.C., Schlicher, K. D., Maier, G. W., Schalkwijk, L., Waßmuth, J., Essig, K., \& Schack, T. (2020). AVIKOM: Towards a mobile audiovisual cognitive assistance system for modern manufacturing and logistics. In F. Makedon (Hrsg.), Proceedings of the 13th ACM international conference on PErvasive technologies related to assistive environments (S. 1-8). : ACM. https://doi.org/10.1145/3389189.3389191.

Nübold, A., Dörr, S.L., \& Maier, G.W. (2015). Considering the orphan: personal identification and its relations with transforma- tional leadership, trust, and performance in a three-path mediation model. Leadership, 11(2), 230-254. https://doi.org/10.1177/ 1742715014522679.

Ometov, A., Shubina, V., Klus, L., Skibińska, J., Saafi, S., Pascacio, P., Flueratoru, L., Quezada Gaibor, D., Chukhno, N., Chukhno, O., Ali, A., Channa, A., Svertoka, E., Qaim, W. B., Casanova-Marqués, R., Holcer, S., Torres-Sospedra, J., Casteleyn, S., Ruggeri, G., Araniti, G., Burget, R., Hosek, J., \& Lohan, E. S. (2021). A survey on wearable technology: History, state-of-the-art and current challenges. Computer Networks. https://doi.org/10.1016/ j.comnet.2021.108074. Artikel 108074.

O'Reilly, C. A., \& Tushman, M.L. (2013). Organizational ambidexterity: past, present, and future. Academy of Management Perspectives, 27(4), 324-338. https://doi.org/10.5465/amp.2013.0025.

O'Reilly, C.A., \& Tushman, M.L. (2016). Lead and disrupt: How to solve the innovator's dilemma. Stanford: Stanford University Press.

Ötting, S. K., \& Maier, G. W. (2018). The importance of procedural justice in Human-Machine Interactions: intelligent systems as new decision agents in organizations. Computers in Human Behavior, 89, 27-39. https://doi.org/10.1016/j.chb.2018.07.022.

Ötting, S. K., \& Maier, G. W. (2021). When artificial intelligence allocates tasks at work: justice of decision procedures and the role of perceived responsibility of the decision agent. Manuskript eingereicht zur Publikation.

Ötting, S. K., Masjutin, L., Steil, J. J., \& Maier, G. W. (2020)a. Let's work together: a meta-analysis on robot design features that enable successful human-robot interaction at work. Human Factors. https://doi.org/10.1177/0018720820966433.

Ötting, S. K., Masjutin, L., \& Maier, G.W. (2020)b. The future of leadership: How is leadership in SMEs going to change?. Manuskript eingereicht zur Publikation.

Palmarini, R., Erkoyuncu, J. A., Roy, R., \& Torabmostaedi, H. (2018). A systematic review of augmented reality applications in maintenance. Robotics and Computer-Integrated Manufacturing, 49, 215-228. https://doi.org/10.1016/j.rcim.2017.06.002.

Paruzel, A., Bentler, D., Schlicher, K., Nettelstroth, W., \& Maier, G.W. (2020). Employee first, technology second: Implementation of smart glasses in a manufacturing company. Zeitschrift für Arbeits- und Organisationspsychologie, 64(1), 46-57. https://doi. org/10.1026/0932-4089/a000292.

Petry, T. (2016). Digital Leadership - Unternehmens- und Personalführung in der Digital Economy. In T. Petry (Hrsg.), Digital Leadership. Erfolgreiches Führen in Zeiten der Digital Economy (S. 21-82). Freiburg: Haufe.

Pfaffinger, K. F., Reif, J. A. M., Spieß, E., \& Berger, R. (2020). Anxiety in a digitalised work environment. Gruppe.Interaktion.Organisation. (GIO), 51, 25-35. https://doi.org/10.1007/s11612-02000502-4.

Podsakoff, P.M., MacKenzie, S.B., Moorman, R.H., \& Fetter, R. (1990). Transformational leader behaviors and their effects on followers' trust in leader, satisfaction, and organizational citizenship behaviors. Leadership Quarterly, 1(2), 107-142. https://doi. org/10.1016/1048-9843(90)90009-7.

Preusser, I., \& Bruch, H. (2014). Leadership 2.0 - Führung in digitalen Zeiten: Leadership-Chancen und Herausforderungen der Digitalisierung. In P. Mehlich, T. Brandenburg \& M. T. Thielsch (Hrsg.), Praxis der Wirtschaftspsychologie III (S. 25-50). Münster: MVWissenschaft.

Sarges, W. (2006). Competencies statt Anforderungen - nur alter Wein in neuen Schläuchen? In H.-C. Riekhof (Hrsg.), Strategien der Personalentwicklung (S. 133-148). Wiesbaden: Gabler.

Schein, E.H., \& von Ameln, F. (2019). Relationships as a key to change. Ed Schein on the legacy and future of Change Management. Gruppe.Interaktion.Organisation. (GIO), 50, 141-144. https://doi.org/10.1007/s11612-019-00462-4. 
Schippmann, J. S., Ash, R. A., Battista, M., Carr, L., Eyde, L. D., Hesketh, B., Kehoe, J., Pearlman, K., Prien, E.P., \& Sanchez, J. I. (2000). The practice of compentency modeling. Personnel Psychology, 53(3), 703-740. https://doi.org/10.1111/j.1744-6570. 2000.tb00220.x.

Schlicher, K., Bentler, D., Paruzel, A., \& Maier, G.W. (in Druck). Arbeit4.0@Hettich: Berufliche Handlungskompetenz in der Umsetzung des Auftragsdurchlaufs von morgen. In R. Dumitrescu (Hrsg.), Gestaltung digitalisierter Arbeitswelten. Handlungsfelder und Praxisbeispiele zur Umsetzung digitalisierter Arbeit. Berlin: Springer.

Schlicker, N., Langer, M., Ötting, S., Baum, K., König, C. J., \& Wallach, D. (2021). What to expect from opening up 'Black Boxes'? Comparing perceptions of justice between human and automated agents. Computers in Human Behavior. https://doi.org/10.1016/j. chb.2021.106837. Artikel 106837.

Schmidt-Huber, M. (2011). Die Entwicklung und Validierung eines Kompetenzmodells und Instruments zur Erfassung von effektivem Führungsverhalten. Bielefeld: Bielefeld University.

Schmidt-Huber, M., Dörr, S.L., \& Maier, G. W. (2014). Die Entwicklung und Validierung eines evidenzbasierten Kompetenzmodells effektiver Führung (LEaD: Leadership Effectiveness and Development). Zeitschrift für Arbeits- und Organisationspsychologie, 58(2), 80-94. https://doi.org/10.1026/0932-4089/a000138.

Schwarzmüller, T., Brosi, P., \& Welpe, I. M. (2015). Führung im digitalen Zeitalter. In T. Becker \& C. Knop (Hrsg.), Digitales Neuland (S. 155-166). Wiesbaden: Springer Gabler. https://doi.org/ 10.1007/978-3-658-09692-2 12

Schwarzmüller, T., Brosi, P., \& Welpe, I. M. (2016). Die veränderte Rolle der Führungskraft im demokratischen Unternehmen. perspektiv:wechsel, 2, 18-19.

Seeber, I., Bittner, E., Briggs, R. O., de Vreede, T., de Vreede, G.J., Elkins, A., Maier, R., Merz, A.B., Oeste-Reiß, S., Randrup, N., Schwabe, G., \& Söllner, M. (2020). Machines as teammates: a research agenda on $\mathrm{AI}$ in team collaboration. Information \& Management, 57(2), 103174. https://doi.org/10.1016/j.im. 2019.103174

Steil, J. J., \& Maier, G. W. (2017). Robots in the digitalized workplace. In G. Hertel, D. Stone, R. Johnson \& J. Passmore (Hrsg.), The Wiley Blackwell Handbook of the psychology of the internet at work (S. 403-422). Chichester: Wiley-Blackwell.

Steil, J. J., \& Maier, G. W. (2020). Kollaborative Roboter. Universale Werkzeuge in der digitalisierten und vernetzten Arbeitswelt. In G.W. Maier, G. Engels \& E. Steffen (Hrsg.), Handbuch Gestaltung digitaler und vernetzter Arbeitswelten (S. 323-346). Berlin: Springer. https://doi.org/10.1007/978-3-662-52979-9_15.

Steinmann, B., Dörr, S. L., Schultheiss, O. C., \& Maier, G. W. (2015). Implicit motives and leadership performance revisited: what constitutes the leadership motive pattern? Motivation and Emotion, 39, 167-174. https://doi.org/10.1007/s11031-014-9458-6.

Steinmann, B., Ötting, S. K., \& Maier, G. W. (2016). Need for affiliation as a motivational add-on for leadership behaviors and managerial success. Frontiers in Psychology, 7, 1972. https://doi.org/10. 3389/fpsyg.2016.01972.

Steinmann, B., Kleinert, A., \& Maier, G. W. (2020). Promoting the underestimated: a vignette study on the importance of the need for affiliation to successful leadership. Motivation and Emotion, 44, 641-656. https://doi.org/10.1007/s11031-020-09833-7.

Tett, R. P., Guterman, H. A., Bleier, A., \& Murphy, P. J. (2000). Development and content validation of a ,hyperdimensional“ taxonomy of managerial competence. Human Performance, 13(3), 205-251. https://doi.org/10.1207/S15327043HUP1303_1.

Welpe, I. M., Brosi, P., \& Schwarzmüller, T. (2018). Digital work design. Frankfurt: Campus.

Wesche, J.S., \& Sonderegger, A. (2019). When computers take the lead: The automation of leadership. Computers in Human Behavior, 101, 197-209. https://doi.org/10.1016/j.chb.2019.07.027.
Westhoff, K., Hagemeister, C., Kersting, M., Lang, F., Moosbrugger, H., Reimann, G., \& Stemmler, G. (Hrsg.). (2005). Grundwissen für die berufsbezogene Eignungsbeurteilung nach DIN 33430 (2. Aufl.). Lengerich: Pabst Science.

Zolfagharifard, E. (19. Mai 2014). Would you take orders from a ROBOT? An artificial intelligence becomes the world's first company director. https://www.dailymail.co.uk/sciencetech/article2632920/Would-orders-ROBOT-Artificial-intelligence-world-scompany-director-Japan.html.

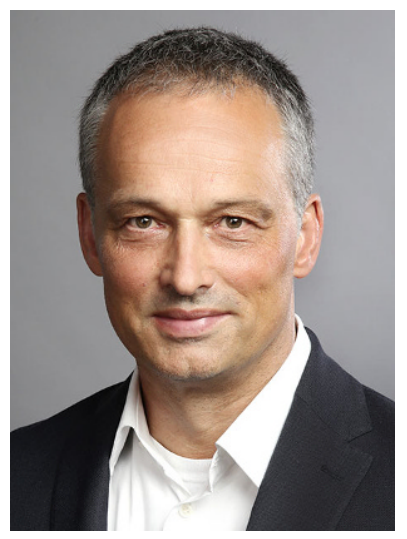

Dr. Stefan Dörr ist Wirtschaftsund Organisationspsychologe und geschäftsführender Partner bei A47 Consulting. Nach seinem Studium der Psychologie in München war er fast 10 Jahre in einem internationalen Konzern der Finanzdienstleistungsbranche für Change Management, Führungskräfte-Entwicklung, Potenzial-AssessmentCenter und Post-Merger-Integration-Prozesse verantwortlich. 1999 gründete er mit zwei Partnern die Beratungsfirma A47-Consulting für Unternehmensentwicklung und Managementdiagnostik. Seine Beratungsschwerpunkte liegen heute in den Bereichen Führungsentwicklung, Changemanagement, Performance Management sowie Managementdiagnostik und -coaching. Er lehrte als Professor für Wirtschaftspsychologie an der Hochschule für angewandtes Management in Erding und forscht im Bereich der Führungsmotivation und transformationalen Führung.

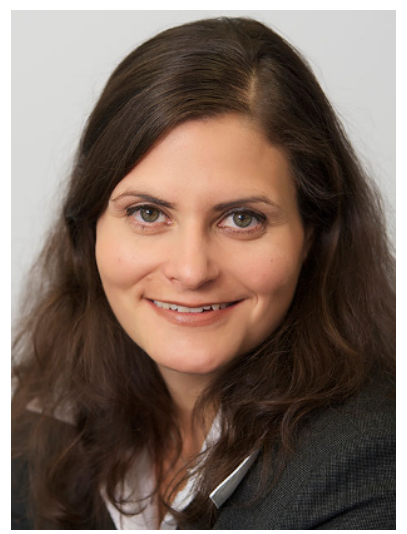

Dr. Marion Schmidt-Huber ist Beraterin bei A47 Consulting in München und wissenschaftliche Mitarbeiterin und Trainerin am Center for Leadership and People Management der LMU München. Nach ihrem Studium der Betriebswirtschaft mit dem Schwerpunkt Bankwesen an der Berufsakademie in Heidenheim studierte sie Psychologie an den Universitäten Trier und Mannheim.

Seit 2007 arbeitet sie bei A47 Consulting vor allem in den Bereichen der Managementdiagnostik, Personal- und Führungskräfteentwicklung. In der Forschung beschäftigt sie sich mit Themenstellungen zu effektivem Führungsverhalten, Führungskompetenzen sowie zur Führungskräfteentwicklung und zu Feedbackprozessen. 


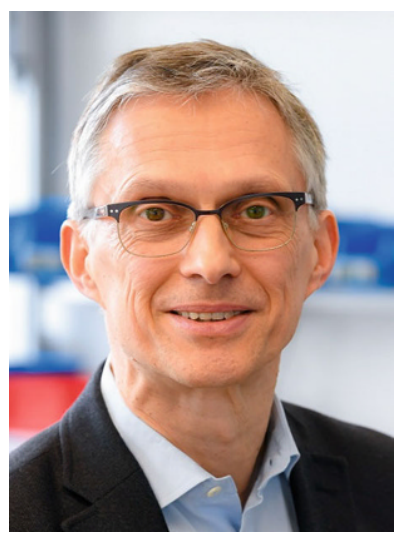

Prof. Dr. Günter W. Maier ist Professor am Lehrstuhl für Arbeitsund Organisationspsychologie der Universität Bielefeld sowie stellvertretender Sprecher des Forschungsschwerpunkts „Digitale Zukunft“ und des NRW Forschungskollegs „Gestaltung von flexiblen Arbeitswelten“. Seine Forschungsinteressen liegen in den Bereichen Gerechtigkeit in der Mensch-MaschineInteraktion, Qualifizierungsbedarf, Führung und Gestaltung von Arbeit in der digitalen Transformation sowie Kreativität. 